\title{
Vibrotactile working memory as a model paradigm for psychology, neuroscience, and computational modeling
}

\section{Tyler D. Bancroft, William E. Hockley and Philip Servos*}

Department of Psychology, Wilfrid Laurier University, Waterloo, ON, Canada

*Correspondence: pservos@wlu.ca

Generalizing the study of short-term memory across different species and paradigms (e.g., behavioral, neuroimaging, etc.) can be challenging. Studies of human memory have often focused on stimuli with substantial semantic content, which are both difficult to model computationally (at least, using biologically based models) and often impossible to convert to animal studies. In contrast, paradigms used in animal studies are sometimes too simple or insufficiently challenging to prove useful in human studies. However, recent theoretical and experimental advances have identified an experimental paradigm that is well suited to both animal, human, and computational research. Vibrotactile working memory has several properties that make it highly suitable for use as a model system: A welldefined, relatively simple neural code, straightforward experimental designs that can be translated from animal to human research (or vice versa) with little change, simple and inexpensive experimental apparatus, and similar neural correlates in both humans and animal models.

As might be deduced from the name, vibrotactile working memory is working memory for vibrational stimuli applied to the hand, most commonly to the dominant index finger. The most common experimental design is the delayed match-to-sample task, in which subjects are presented with a stimulus to be remembered (the target), an unfilled delay period, followed by a second stimulus (the probe), of the same frequency or of a different frequency as the target stimulus, and are asked to decide if the target and probe are of the same frequency or different frequencies. Notably, these vibrational stimuli are non-semantic, allowing use of the same experimental designs in both humans and non-humans. While it is theoretically possible that human subjects are verbally labeling the stimuli and storing that label, thereby converting the task from a vibrotactile to a verbal working memory task, there is no evidence of subjects using such a strategy. There has been no activation of language-specific cortical regions found in a number of human imaging studies (Sörös et al., 2007; Spitzer et al., 2010). Further, a recent human behavioral study implicitly tested this hypothesis, and found no evidence of verbal coding (Bancroft et al., under review).

Research in both humans and nonhuman primates has identified a set of four regions critical for vibrotactile working memory: primary somatosensory cortex (SI), secondary somatosensory cortex (SII), medial premotor cortex (MPC), and prefrontal cortex (PFC; Romo and Salinas, 2003). Extensive single-cell recording work in macaques has been done by Romo et al. (1999) allowing the tentative assignment of roles to these regions: SI is believed to be involved in stimulus processing, SII in stimulus processing and decision-making, PFC in stimulus storage and decisionmaking, and MPC in converting decisions into motor responses. Functional MRI (Preuschhof et al., 2006; Sörös et al., 2007; Hegner et al., 2010), EEG (Spitzer et al., 2010), and MEG (Haegens et al., 2010) research in humans has produced results that are generally consistent with single-cell recordings in macaques, suggesting there is substantial similarity between the neural correlates of vibrotactile working memory in human and non-human primates.

The vibrational frequency of stimuli is encoded in the firing rates of neurons, with firing rates being monotonic functions of the stimulus frequency. This relatively simple relationship between firing rate and stimulus frequency is not only convenient for modelers, but also allows the extraction of useful information from experimental data. Previous single-cell recording research has used information-theoretic methods to determine when neuronal firing rates are carrying information about stimulus frequency (Romo et al., 1999; Hernández et al., 2002; Romo and Salinas, 2003). More recently, Spitzer et al. (2010) and Spitzer and
Blankenburg (2011) were able to extract the frequency of a stored stimulus by observing modulations of beta-band (20-25 Hz) EEG activity in human PFC during vibrotactile working memory tasks. The relatively straightforward neural code used to encode and store vibrotactile stimuli allows examination of the flow of information through the neural systems involved in working memory, and also allows disambiguation between systems involved in stimulus processing and storage, and systems involved in other cognitive functions, such as attention or motor functioning related to response.

On a cognitive level, vibrotactile working memory shares many traits with other domains of working memory, including susceptibility to interference (Harris et al., 2001; Bancroft and Servos, 2011; Bancroft et al., 2011), a storage capacity of more than one item (Bancroft et al., under review), and demand on attentional systems (Hannula et al., 2010; Spitzer and Blankenburg, 2011). Further, recent EEG research has shown that subjects are able to remove individual stimuli from a stored set of stimuli when so instructed (Spitzer and Blankenburg, 2011). As vibrotactile working memory shares common qualities with other working memory systems, but does not contain semantic content, it is useful for testing theories of working memory, as we have access to both human and animal research. For example, Postle (2006) suggested that sensory cortex may be a storage substrate for working memory. However, information-theoretic analyses of single-cell recordings in primary and secondary somatosensory cortex suggest that stimuli are not represented in these areas during the delay period, but rather in non-sensory regions of PFC (Romo et al., 1999; Hernández et al., 2002; Romo and Salinas, 2003). Similarly, research into vibrotactile working memory has begun to pose problems for the venerable multiple-components model (see Repovš and Baddeley, 2006 for a recent overview of this model). The multiple-components model postulates 
modality-specific components involved in the storage and processing of stimuli (for example, the visuospatial sketchpad). The present incarnation of the model, however, does not contain a component capable of storing vibrotactile stimuli, nor do the neural correlates proposed for those components overlap substantially with the neural correlates of vibrotactile working memory. Thus, findings associated with vibrotactile working memory pose problems for the generality of the multiple-components model.

Experimental apparatus used in vibrotactile working memory tasks can be constructed inexpensively and with relative ease. In our lab, we use devices constructed by mounting a large nylon screw on the middle of a speaker cone, and mounting the cone inside a plastic housing such that the top of the screw is flush with the housing. The speaker is connected to a computer's standard headphone jack, and driven by a sine wave of the desired frequency. Subjects place their index finger on the surface of the screw, which then vibrates when the speaker vibrates. Such devices are simple to construct, and can be built using offthe-shelf components. Standard experimental software can be used to deliver stimuli. Stimulators using piezoelectric devices or solenoids to deliver vibrations are also available (but proportionately more expensive). Such devices, however, are not necessary in most cases, and one can construct a perfectly serviceable device at a reasonable cost.
Vibrotactile working memory is a paradigm that offers researchers the ability to draw on the human, animal, and computational literatures simultaneously. Research translates well between human and nonhuman subjects, and vibrotactile memory relies on a well-defined neural code and set of cortical regions, making it an ideal model system that can be studied using behavioral, imaging, and computational paradigms.

\section{REFERENCES}

Bancroft, T., and Servos, P. (2011). Distractor frequency influences performance in vibrotactile working memory. Exp. Brain Res. 208, 529-532.

Bancroft, T. D., Servos, P., and Hockley, W. E. (2011). Mechanisms of interference in vibrotactile working memory. PLoS ONE 6, e22518. doi: 10.1371/journal. pone. 0022518

Haegens, S., Osipova, D., Oostenveld, R., and Jensen, O. (2010). Somatosensory working memory performance in humans depends on both engagement and disengagement of regions in a distributed network. Hum. Brain Mapp. 31, 26-35.

Hannula, H., Neuvonen, T., Savolainen, P., Hiltunen, J., Ma, Y., Antila, H., Salonen, O., Carlson, S., and Pertovaara, A. (2010). Increasing top-down suppression from prefrontal cortex facilitates tactile working memory. Neuroimage 49, 1091-1098.

Harris, J. A., Harris, I. M., and Diamond, M. E. (2001). The topography of tactile working memory. J. Neurosci. 21, 8262-8269.

Hegner, Y., Lee, Y., Grodd, W., and Braun, C. (2010). Comparing tactile pattern and vibrotactile frequency discrimination: a human fMRI study. $J$. Neurophysiol. 103, 3115-3122.

Hernández, A., Zainos, A., and Romo, R. (2002). Temporal evolution of a decision-making process in medial premotor cortex. Neuron 33, 959-972.
Postle, B. (2006). Working memory as an emergent property of the mind and brain. Neuroscience 139, 23-38.

Preuschhof, C., Heekeren, H., Taskin, B., Schubert, T., and Villringer, A. (2006). Neural correlates of vibrotactile working memory in the human brain. J. Neurosci. 20, 13231-13239.

Repovš, G., and Baddeley, A. (2006). The multi-component model of working memory: explorations in experimental cognitive psychology. Neuroscience 139, 5-21.

Romo, R., Brody, C. D., Hernández, A., and Lemus, L. (1999). Neuronal correlates of parametric working memory in the prefrontal cortex. Nature 339, 470-473.

Romo, R., and Salinas, E. (2003). Flutter discrimination: neural codes, perception, memory, and decision making. Nat. Rev. Neurosci. 4, 203-218.

Sörös, P., Marmurek, J., Tam, F., Baker, N., Staines, W. R., and Graham, S. J. (2007). Functional MRI of working memory and selective attention in vibrotactile frequency discrimination. BMC Neurosci. 8, 48. doi: 10.1186/1471-2202-8-48

Spitzer, B., and Blankenburg, F. (2011). Stimulusdependent EEG activity reflects internal updating of tactile working memory in humans. Proc. Natl. Acad. Sci. U.S.A. 108, 8444-8449.

Spitzer, B., Wacker, E., and Blankenburg, F. (2010). Oscillatory correlates of vibrotactile frequency processing in human working memory. J. Neurosci. 30, 4496-4502.

Received: 06 October 2011; accepted: 21 November 2011; published online: 09 December 2011.

Citation: Bancroft TD, Hockley WE and Servos $P$ (2011) Vibrotactile working memory as a model paradigm for psychology, neuroscience, and computational modeling. Front. Hum. Neurosci. 5:162. doi: 10.3389/ fnhum.2011.00162

Copyright (c) 2011 Bancroft, Hockley and Servos. This is an open-access article distributed under the terms of the Creative Commons Attribution Non Commercial License, which permits non-commercial use, distribution, and reproduction in other forums, provided the original authors and source are credited. 